\title{
Surface Kinetics of Laser-Atomic Layer Epitaxy (Laser-ALE) of GaAs, AlAs and AlGaAs
}

\author{
Yoshinobu AOYAGI*, Sohachi IwAI and Takashi MEGURo
}

Received September 19, 1991; Accepted September 24, 1991

\begin{abstract}
The mechanism of the laser assisted atomic layer epitaxy (laser-ALE) is studied for the purpose of the strict structural control of thin epitaxial layers of alloy semiconductors. The photochemical "site-selective-decomposition" of metalorganic compounds at the As atomic layer is a main mechanism for the laser ALE. Applications of the laser ALE to a line epitaxy and a quantum well structure are also described.
\end{abstract}

\section{INTRODUCTION}

The growth of III-V compound semiconductors by the metalorganic vapor-phase epitaxy (MOVPE) has much attention due to its potentiality for a wide variety of the optoelectronic device using alloy semiconductors. There are many advantages in low-temperature processes for the fabrication of well-defined structural devices with precise control. The new method for the epitaxial growth of the atomic layer epitaxy ${ }^{1)}$ (ALE) is an attractive method for the possibility to reduce the growth temperature with strict structural control. Several studies on the ALE have been demonstrated in recent years ${ }^{2 \sim 7)}$. To achieve the ALE, in which self-limiting mechanism for each monolayer growth at the $100 \%$ surface coverage is required, an excimer ${ }^{2)}$ or an $\mathrm{Ar}^{+}$laser $^{7}$ has been used in metal organic- $\mathrm{AsH}_{3}$ system for GaAs crystal growth. This laserassisted crystal growth is also used in a conventional metal organic vapor phase epitaxy (MOVPE) to get a patterned crystal growth ${ }^{8 \sim 10)}$. However, the mechanism of the photo-irradiation effects on the crystal growth has not yet been clarified.

The Institute of Physical and Chemical Research (Hirosawa, Wako, Saitama 351-01)

Key Words: Laser ALE, GaAs, Site-Selective Decomposition ALE, GaAlAs, AlAs
In the conventional photochemical vapor deposition (photo-CVD) by the visible laser, such as direct writing CVD of a metal wire on $\mathrm{Si}$ by an $\mathrm{Ar}^{+}$laser ${ }^{10)}$, photo thermal decomposition of the source gases is considered to be important. However, in the photo-assisted crystal growth of GaAs by the visible laser, the photochemical surface effect seems to be an important mechanism ${ }^{7 \sim 9)}$, though optical absorption in the visible region does not exist in $\mathrm{AsH}_{3}$ and metalorganic compounds such as triethylgallium (TEG) and triethylaluminum (TEA). Therefore, it is very interesting to clarify whether the visible laser irradiation effects are photothermal effects or photochemical effects.

In this report we discuss the mechanism of the laser-ALE of GaAs, AlAs and AlGaAs by SLMOVPE technique. We emphasize that the photo-selective chemical decomposition of TEG or TEA at the As atomic surface is an important mechanism for the GaAs epitaxial growth under the laser irradiation.

\section{EXPERIMENTAL PROCEDURES}

In our laser ALE, the $\mathrm{AsH}_{3}$ and TEG/TEA were alternatively supplied into the growth chamber during $1 \mathrm{sec}$ with $3 \mathrm{sec}$ time interval between them and the $\mathrm{As}$ and $\mathrm{Ga} / \mathrm{Al}$ atomic layer is alternatively grown on the (100) GaAs 


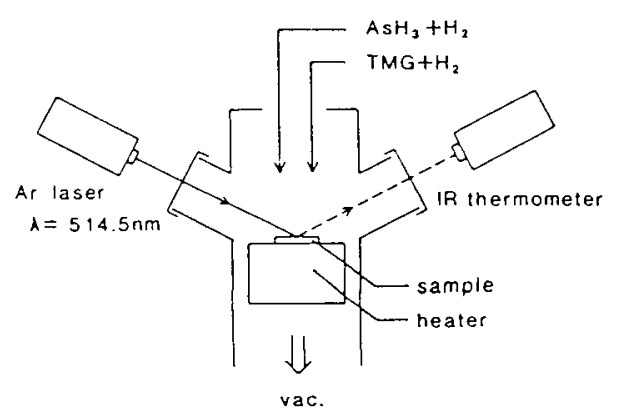

Fig. 1 Schematic diagram of the experimental system.

substrate. The experimental system is shown in Fig. 1 schematically. $\mathrm{H}_{2}$ gas was continuously flowed into the chamber at a rate of $2700 \mathrm{sccm}$ to ensure purging of residual gases of $\mathrm{AsH}_{3}$ and TEG/TEA. The GaAs substrate was irradiated by the $\mathrm{Ar}^{+}$laser or the YAG laser during $1 \mathrm{sec}$ at various wavelengths of the laser light. Details of experimental procedures and the experimental conditions have been shown in the previous paper $^{n}$.

\section{RESULTS AND DISCUSSION}

\subsection{GaAs ALE}

To achieve the ALE it is essential to introduce the self-limiting mechanism for each layer growth at the $100 \%$ surface coverage. In case of the As atomic layer, one monolayer growth is easily achieved under a proper growth condition for a reason of the high vapor pressure of As. However, in case of the $\mathrm{Ga}$ atomic layer it is difficult to achieve the monolayer growth for the low vapor pressure of $\mathrm{Ga}$. That is, if the Ga gas source of more than the quantity needed for the $100 \%$ surface coverage is decomposed on the GaAs surface the excess Ga does not desorb and the monolayer growth condition is not achieved. In the laser ALE the laser is used for achieving the self-limiting decomposition of the source gas for the $\mathrm{Ga}$ atomic layer.

Figure 2 shows a variation of growth rate of $\mathrm{GaAs}$ as a function of the laser power density absorbed into the substrate at $350^{\circ} \mathrm{C}$ of growth temperature. Open circles, triangles and squares denote growth rates under UV, visible and IR

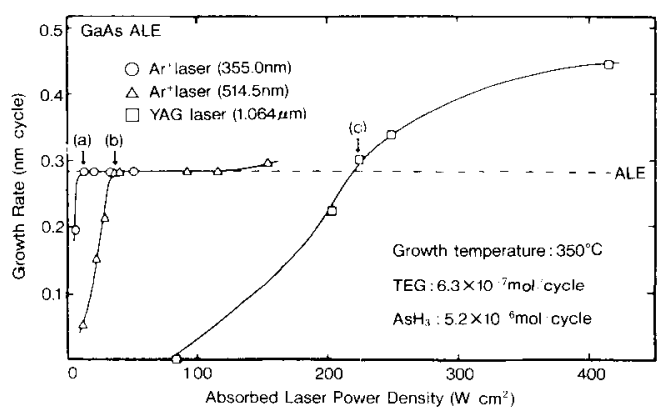

Fig. 2 Growth rate of GaAs vs. the laser power density.

laser irradiation, respectively. The absorbed laser power density was estimated as a difference between the intensities of the incident beam and the reflected beam. Growth rates for the UV and visible laser irradiation is independent of the incident laser power for the density of more than 13 and $36 \mathrm{~W} / \mathrm{cm}^{2}$, respectively, while the growth rate with the IR laser irradiation increases gradually with increasing the laser power density in whole region of the laser power and exhibits no plateau. For UV laser the threshold laser power density for the ALE is much lower than that in the case of the visible laser irradiation. In these plateau region the ALE has been achieved ${ }^{6,7)}$.

Figure 3 shows a temperature dependence of growth rate at each threshold laser power density as shown as (a), (b) and (c) in Fig. 2. In the case of the IR laser irradiation, the laser power density at the growth rate of the ALE was adopted as the threshold value, since the ALE was not achieved and an obvious threshold of laser power density could not be identified. The growth rates of the pulsed MOVPE without the laser irradiation are also shown by solid circles for the comparison. With the UV or visible laser irradiation, the growth rate exhibits a plateau between $350 \sim 380^{\circ} \mathrm{C}$ of growth temperature. The growth rate with the IR laser irradiation reveal the similar tendency as the case of thermal epitaxy, but shifted toward low temperature region. This is considered to be caused from increase of the substrate temperature by the laser irradiation. For the IR laser irradiation the epitaxial region is a semispherical spot which well-reflected 


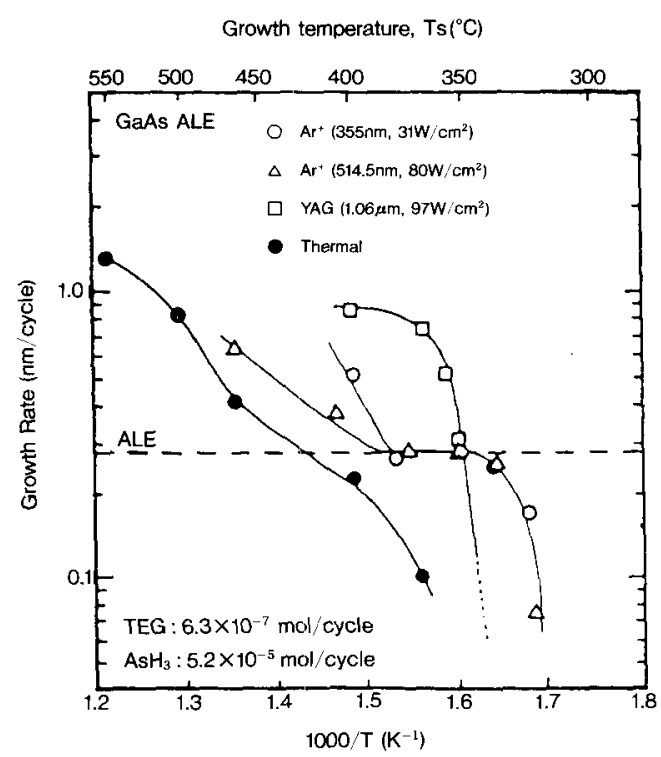

Fig. 3 Growth rate of GaAs vs. growth temperature.

gaussian profile of the laser as same as in the case of ordinary laser-assisted deposition. In the case of the UV or visible laser irradiation the ALE is achieved and a plateau-shaped spot with smooth surface morphology which is independent of the laser gaussian profile is obtained.

\subsection{AlAs ALE}

Figure 4 (a) shows an optical micrograph of the typical feature of growth region grown on the AlAs buffer layer. It is clear that the plateaushaped epitaxial region was obtained from the interference microscopic observation as shown in Fig. 4 (b) and that the gaussian distribution of the laser beam did not reflect on the epitaxial shape. The similar tendency was obtained in the case of the growth on the GaAs substrate.

Figure 5 indicates the dependence of the growth rate on the laser power density at $350^{\circ} \mathrm{C}$ grown on the GaAs substrate. As shown in the inserted figure, $\Delta d$ and $d+\Delta d$ (total) correspond to the growth rate with and without the laser irradiation, respectively. The growth rate gradually increased below $260 \mathrm{~W} / \mathrm{cm}^{2}$ and was independent of the laser power density above 260 $\mathrm{W} / \mathrm{cm}^{2}$. Additionally, in the case of the growth on the AlAs buffer layer, there was no obvious

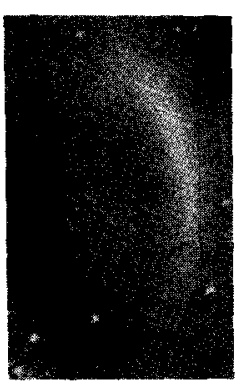

(a) $0.1 \mathrm{~mm}$

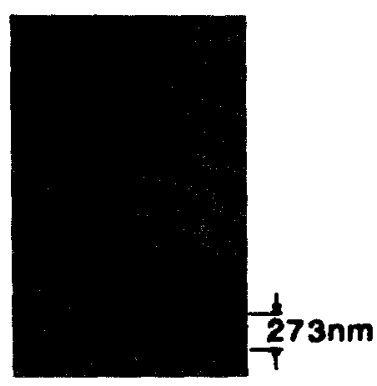

(b)
Fig. 4 (a) Optical micrograph of typical epitaxial region and (b) the interference microscopic observation of the same area.

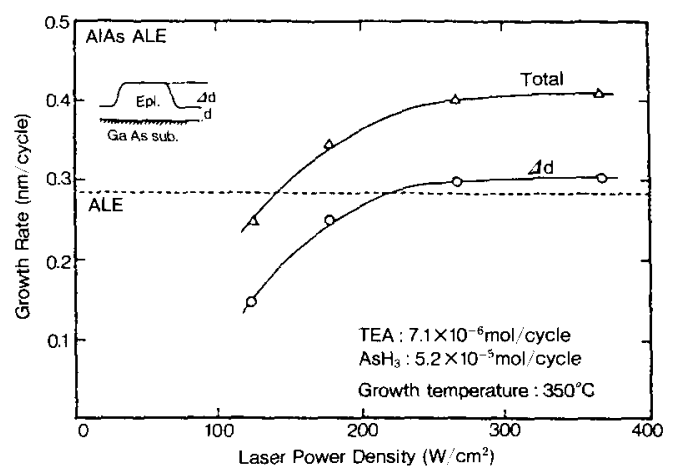

Fig. 5 Growth rate of AlAs vs. the laser power density,

change on the growth rate compared with the growth on the GaAs substrate under the present experimental condition. It is found that the laser power density for the laser-ALE of AlAs was several times larger than that of GaAs using TEG.

\subsection{AlGaAs ALE}

Figure 6 shows the growth rate of AlGaAs as a function of TEG flux under the constant TEA flux condition at $360^{\circ} \mathrm{C}$. Solid circles indicate the growth rate of $\mathrm{AlGaAs}$ and, as the comparison, open circles show the TEG flux dependence of the growth rate of GaAs. While in the case of the growth of GaAs the ALE was achieved around $1 \times 10^{-7} \mathrm{~mol} / \mathrm{cycle}$ of TEG flux, the amount of TEG for the ALE is increased in the case of AlGaAs with a coincidental TEA feeding. It is considered that the adsorption of TEG at the suitable surface site was restricted by adsorbed TEA molecules. In addition, it is expected that 


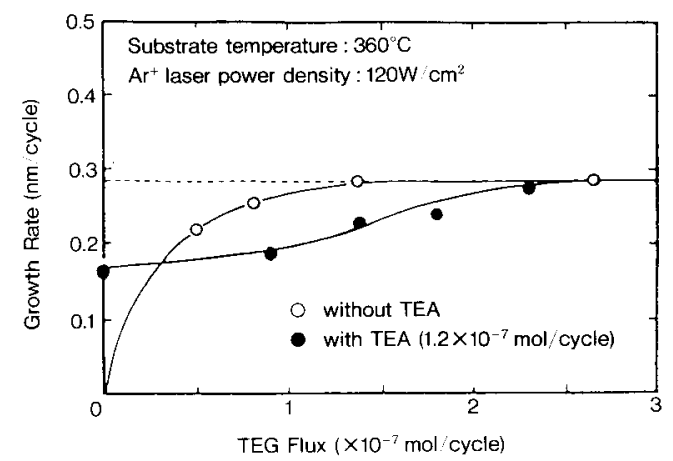

Fig. 6 Growth rate of AlGaAs vs. TEG flux. Open circle shows the TEG flux dependence of the growth rate of $\mathrm{GaAs}$ as a reference.

the $\mathrm{Al} / \mathrm{Ga}$ ratio in the epitaxial region grown with the laser irradiation is less than that in the region grown without the laser irradiation, because the enhancement ratio of TEA decomposition due to the laser irradiation is less than that in the case of TEG, which can be wellunderstood from the results of the AlAs ALE shown in the section 3.2.

\section{GROWTH MECHANISM OF THE LASER ALE}

We propose from above discussion the schematics for the surface decomposition processes in the case of the ALE and the ordinal pulsed MOVPE as shown in Fig. 7 (a) and (b), respectively. Fig. 7 (a) shows the surface process in the laser-ALE and we defined as the "siteselective-decomposition" process which explains the experimental results. For this "site-selectivedecomposition" model, TEG molecules easily decompose only on As atoms, so that the ideal ALE can be obtained. On the other hand, in the case of the ordinary pulsed MOVPE as shown in Fig. 7 (b) which also reveals the case for the IR

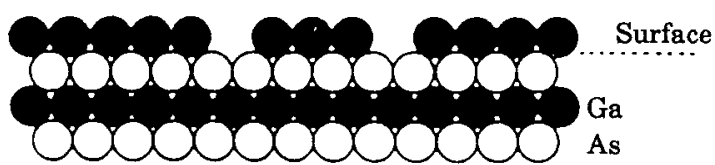

(a) laser irradiation, Ga atoms deposit not only on As atoms but on $\mathrm{Ga}$ atoms. It is, therefore, difficult to obtain the ideal ALE and the surface roughness in the case of the IR laser irradiation is considered to be caused by the growth process without the "site-selective-decomposition".

As the possible mechanism for the "siteselective-decomposition", following two mechanisms are considered.

(1) Photo-adsorption by the surface adsorbed species: Neither TMG nor TEG in gas phase have adsorption in the visible regions. The adsorption spectrum of those metal organic compounds chemically adsorbed on the As surface of GaAs, however, extends to the visible regions and the direct photoadsorption becomes possible for the adsorbed metal organic compounds. Thus, they are decomposed by the photo-absorption selectively on the As surface.

(2) Carrier generation by the substrate photo-absorption: Free carriers generated in the substrate by the photo-absorption diffuse to the substrate surface. The charge transfer to the chemisorbed species on the As surface weaken the Ga-As bond.

To investigate the effects of free carriers generated by photo-absorption, $\mathrm{Ar}^{+}$laser ALE experiments were made for two types of heterostructure substrates: (a) n-AlAs grown on p-GaAs, and (b) p-AlAs grown on n-GaAs. AlAs is transparent for $\mathrm{Ar}^{+}$laser light. So, carriers are generated in the GaAs under the AlAs layer. In the structure (a) and (b), holes and electrons generated in $\mathrm{GaAs}$ do not arrive the AlAs surface, respectively. Experimental results have shown that the laser ALE can be attained by both structures. Thus, the mechanism (1) is considered to be dominant. In the case of $\mathrm{GaAs}$ growth by the laser ALE on the AlAs/GaAs

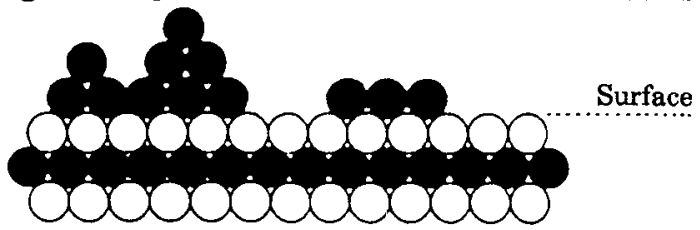

(b)

Fig. 7 Schematic growth procedure (a) for the laser-ALE and (b) for the thermal pulsed MOVPE. 


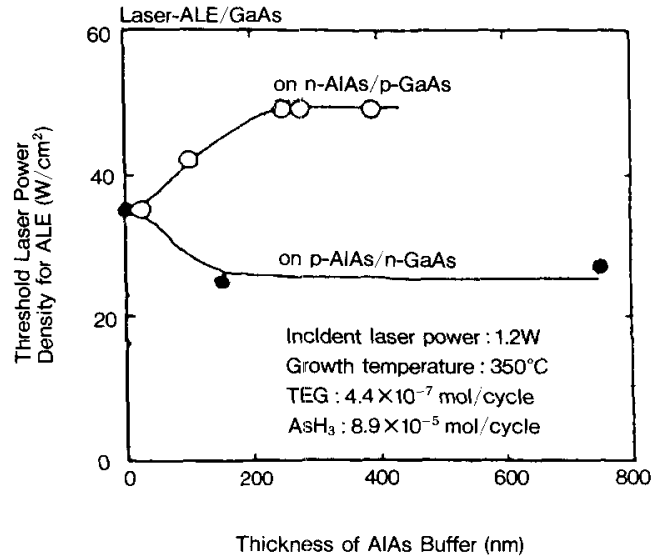

Fig. 8 Threshold laser power density for ALE as a function of AlAs film thickness.

substrates, the threshold laser power density for ALE was found to change slightly depending on the AlAs film thickness as shown in Fig. 8, although the reason is under investigations.

\section{APPLICATIONS OF THE LASER ALE}

The surface of the crystal grown by the laser ALE technique is very flat and by scanning the laser, we can achieve a line epitaxy with a flat surface. In the conventional direct writing photo epitaxy the profile of the epitaxial layer is not flat $^{11)}$ and it is very difficult to construct a heterostructure or a quantum well structure with a control of the thickness, though this control is essential for device fabrication. However, as shown in Fig. 9, the line epitaxy with a flat top surface is easily obtained in our laser ALE by the direct writing technique of the Ar ion laser.

In the line patterning by the laser ALE the crossing part of two lines is also flat in contrast to the line patterning by the conventional photoCVD. In case of the conventional photo-CVD the thickness of the crossed part of two lines are almost twice of the thickness of each line. However, in the laser ALE the thickness is limited by the number of cycles as mentioned previous chapter, and therefore the thickness at the crossing part is as same as that of the line, if

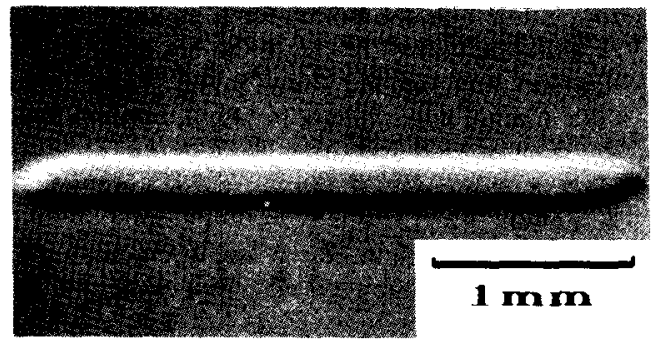

Fig.9 Optical micrograph of the epitaxial line of GaAs grown by the scanning laser-ALE.

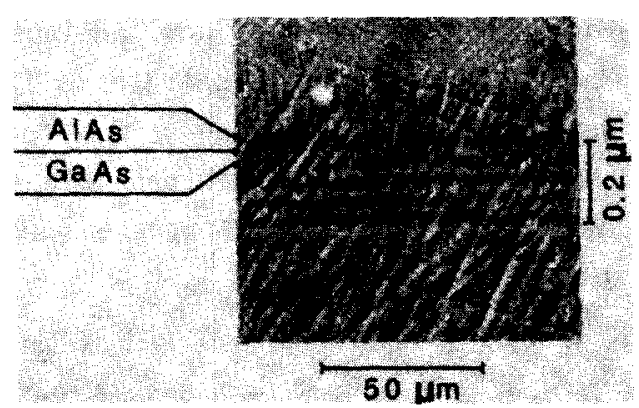

Fig. 10 Cross-section of the quantum well structure grown by the laser-ALE observed by the tilt polishing method.

we write the pattern within the period of the one cycle. This characteristic is a big advantage of the line patterning by the laser ALE.

Figure 10 shows a typical GaAs/AlAs quantum well structure obtained by the laserALE. In this case the number of the atomic layer for GaAs and AlAs is 100. From this result it is certain that we can make the quantum well structure by this laser-ALE technique.

\section{CONCLUSION}

From these experimental results we can conclude that the visible laser irradiation induces the photochemical "site-selective-decomposition" of TEG or TEA at the surface of GaAs but not induce a photothermal decomposition. The laser ALE technique has several superior characteristics for device fabrication. 


\section{REFERENCES}

1) T. Suntola, in Ext. Abstr. Int'l Conf. Solid State Devices and Mater., Kobe, p.647. 1984 (Jpn. Soc. Appl. Phys., Tokyo, 1984); T. Suntola and M. Anston, U. S. Patent, 4,058,130 (1977).

2) J. Nishizawa, Jpn. J. Appl. Phys. Suppl., 19-1, 3 (1980).

3) S. M. Bedair, M. A. Tischler. T. Katsuyama, and N. A. El-Marshy, Appl. Phys. Lett., 47, 51 (1985).

4) N. Kobayashi, T. Makimoto, and Y. Horikoshi, in 12th Int'l Conf. on GaAs and Related compounds, p. 737 (Karuizawa, 1985).

5) A. Usui and H. Sunakawa, in 12th Int'l Conf. on GaAs and Related compounds, p.753
(Karuizawa, 1985).

6) A. Doi, Y. Aoyagi, and S. Namba, Appl. Phys. Lett., 48, 1787 (1986).

7) A. Doi, Y. Aoyagi, and S. Namba, Appl. Phys. Lett., 49, 785 (1986).

8) Y. Aoyagi, S. Masuda, and S. Namba, Appl. Phys. Lett., 47, 95 (1985); Appl. Phys. Lett., 48, 379 (1986).

9) Y. Aoyagi, M. Kanazawa, A. Doi, S. Iwai, and S.Namba, J. Appl. Phys., 60, 3131 (1986).

10) H. Kukimoto, Y. Ban, H. Takechi, and M. Ishizaki, J. Cryst. Growth, 77, 223 (1986).

11) S. M. Bedair, J. K. Whisnant, N. H. Karam, M. A. Tischler, and T. Katsuyama, Appl. Phys. Lett., 46, 174 (1986). 